\title{
Learning from Heterogeneous Networks: Methods and Applications
}

\author{
Chuxu Zhang \\ University of Notre Dame \\ czhang11@nd.edu
}

\begin{abstract}
Complex systems in different disciplines are usually modeled as heterogeneous networks. Different from homogeneous networks or attributed networks, heterogeneous networks are associated with complexity in heterogeneous structure or heterogeneous content or both. The abundant information in heterogeneous networks provide opportunities yet pose challenges for researchers and practitioners to develop customized machine learning solutions for solving different problems in complex systems. We are motivated to do significant work for learning from heterogeneous networks. In this paper, we first introduce the motivation and background of this research. Later, we present our current work which include a series of proposed methods and applications. These methods will be introduced in the perspectives of personalization in web-based systems and heterogeneous network embedding. In the end, we raise several research directions as future agenda.
\end{abstract}

\section{CCS CONCEPTS}

- Information systems $\rightarrow$ Web applications; • Computing methodologies $\rightarrow$ Machine learning; Knowledge representation and reasoning.

\section{KEYWORDS}

Heterogeneous Networks; Personalization; Network Embedding

\section{ACM Reference Format:}

Chuxu Zhang. 2020. Learning from Heterogeneous Networks: Methods and Applications. In The Thirteenth ACM International Conference on Web Search and Data Mining (WSDM'20), February 3-7, 2020, Houston, TX, USA. ACM, New York, NY, USA, 2 pages. https://doi.org/10.1145/3336191.3372182

\section{INTRODUCTION}

In today's information and computational society (Fig. 1), complex systems are often modeled as heterogeneous networks associated with heterogeneous data. The heterogeneous network data provide researchers and practitioners opportunities to understand systems more comprehensively while pose challenges for knowledge discovery from them. The challenges come from not only the complexity and heterogeneity of the data for investigation but also the requirement of the target problem to be solved. Besides the difficulty of extracting information from the complex data, it is hard to fuse the

Permission to make digital or hard copies of part or all of this work for personal or classroom use is granted without fee provided that copies are not made or distributed for profit or commercial advantage and that copies bear this notice and the full citation on the first page. Copyrights for third-party components of this work must be honored

For all other uses, contact the owner/author(s).

WSDM '20, February 3-7, 2020, Houston, TX, USA

(C) 2020 Copyright held by the owner/author(s).

ACM ISBN 978-1-4503-6822-3/20/02.

https://doi.org/10.1145/3336191.3372182

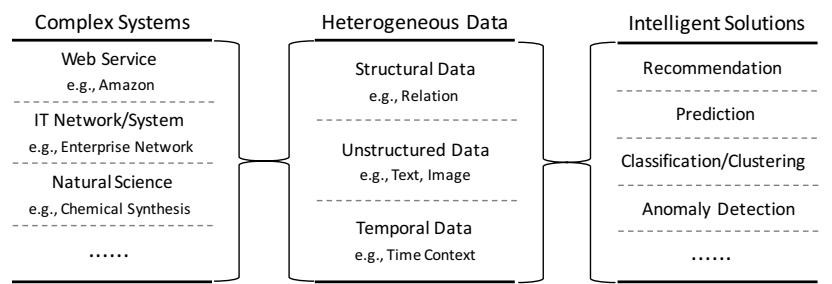

Figure 1: Overview of our research: turning heterogeneous network data into knowledge, upon what developing AI solutions for a diverse set of applications.

extracted knowledge in a unified manner so as to facilitate various applications. Can we develop artificial intelligence (AI) solutions to extract, represent, fuse knowledge from heterogeneous data so as to tackle various challenges in complex systems?

The goal of our research is to harnesses the power of heterogeneous network data, turn them into knowledge, develop AI solutions based on the extracted knowledge for various real-world applications. Successful AI solutions should solve two fundamental research questions brought forth by the above challenges: (1) How to extract and represent useful information from the data of heterogeneous structure, or multi-modal/source, or both? (2) How to fuse the extracted knowledge in customized machine learning models for solving target problems across different disciplines? We have been developing a series of methodologies and algorithms to answer these questions, which have been deployed and validated in several research topics. In the following sections, we will outline our current research and future agenda.

\section{CURRENT RESEARCH}

Nowadays, people are deeply involved in various web services such as social media, online shopping, academic search, etc. Those systems are usually modeled as heterogeneous networks, representing multi-typed nodes interconnected by multi-typed edges as well as multi-modal contents in them. It is hard for individuals to seek personal needs in those systems. Thus developing intelligent solutions to automatically provide personalized services to them is important. In addition, the abundant heterogeneous information in systems requires both a domain understanding and large exploratory search space when doing feature engineering activities of customized machine learning models for different purposes. Therefore, developing intelligent solutions to automate the discovery of feature representation for various tasks is crucial. Accordingly, our research tackles problems in two perspectives: (a) personalization in web-based systems; (b) heterogeneous network embedding.

\subsection{Personalization in Web-based Systems}

The problem of personalization aims at automatically recommending suitable objects (e.g., items) to target entities (e.g., users) in 
the system. As many online web services are modeled as heterogeneous networks, extracting and fusing useful information from such structure would benefit recommender system designs. We have done significant work in this area. We proposed CUNE [10] to leverage user-item bipartite structure to identify similar users of each user, which are incorporated into standard recommendation models (e.g., matrix factorization, pairwise ranking) and improve performances in both rating prediction and item ranking tasks. We further proposed two models, i.e., ImWalkMF [11] for reducing rating prediction error by considering both direct and indirect correlations among users and items, and WalkRanker [4] for elevating item ranking quality by incorporating multiple user-item relations into a unified pairwise ranking model. Moreover, we developed MARank [3] to improve sequential recommendation by unifying both individual- and union-level item sequential correlations in preference ranking model. Besides the recommender system study, we have also finished some other work for personalization in the online academic service systems, which tackle the data of heterogeneous networks with text content in nodes. We proposed Camel [5] for author identification, which is also useful for other tasks such as reviewer recommendation. Camel performs joint optimization of content encoder based distance metric learning and heterogeneous structure based integrative learning. Camel can effectively predict correlated/true authors of anonymous papers. Besides Camel, we also developed TSR [12], a task-guided and semantic-aware ranking model, to effectively recommend academic papers to researchers, especially for whom with little knowledge of the discipline.

\subsection{Heterogeneous Network Embedding}

The purpose of heterogeneous network embedding is to automate the discovery of meaningful vector representation for each node in the network so as to facilitate various downstream tasks. We have pursued this direction and generated several research outputs in this area. We proposed SHNE [8] to learn node embeddings in heterogeneous networks with text information (e.g., academic network with text content in nodes). SHNE addresses the challenge of extracting and fusing both structural closeness and semantic correlations by integrating node content (i.e., text) as a deep encoding function into the heterogeneous structure embedding framework, and elevates performances in various network mining tasks. Following the success of SHNE and in order to achieve a bigger scenario goal that learning node representations in heterogeneous networks with heterogeneous content in nodes (e.g., online shopping networks with description/review text, image, attribute in nodes), we further developed HetGNN [6], a heterogeneous graph neural network framework. HetGNN joints node heterogeneous contents encoding, type-based neighbors aggregation, and heterogeneous types combination. In the training stage, a graph context loss and a mini-batch gradient descent procedure were employed to learn the model parameters. HetGNN has been deployed in a number of web service system data and performs well for various downstream tasks including link prediction, node recommendation, and node classification/clustering. In addition to SHNE and HetGNN, we also proposed FSRL [9], a heterogeneous relation learning model in knowledge graphs. FSRL considers heterogeneity of both neighboring entities and relations in learning target entity's embedding, which elevates link prediction performance.

\section{FUTURE AGENDA}

Our future research will be to develop effective, efficient, and interpretable AI solutions for heterogeneous network data: (1) In-depth analysis. Most of complex systems are associated with temporal data (e.g., time series) which is useful for investigating system dynamics and improving the predictive analysis. We have done some work for time series and spatial-temporal data analysis [1,7], and plan to leverage those information to further improve the stateof-the-art for different problems. (2) Broad applications. We are excited to develop intelligent solutions for various problems in different disciplines such as synthetic chemistry. Many of chemical synthesis problems can be transformed to machine learning tasks in heterogeneous network data. For example, predicting a chemical reaction result is to infer edge change (i.e., bond change) in heterogeneous network (i.e., molecular graph with multi-typed attributed atoms and bonds). (3) Learning with small labeled data. Lacking ground-truth labels is common in heterogeneous network data and it is often expensive to collect such labels. For example, online shopping systems and knowledge graphs face coldstart issue. It is practically significant to create efficient solutions to solve the challenges of small labeled data. Our prior work FSRL [9] and GFL [2] tackles limited supervisory labels in graph data. Depart from these work, we will investigate more techniques for developing efficient solutions using small labeled data. (4) Interpretable learning. The current machine learning algorithms for heterogeneous network data are mostly lack of explainability. For example, the link prediction methods predict connection between two nodes yet do not show why such connection is inferred. Equipping models with explainability will bring benefits in transparency, trustworthiness, and effectiveness. We are interested in leveraging advanced techniques to develop interpretable solutions.

Acknowledgments. This work is supported by the Army Research Laboratory award W911NF-09-2-0053 and the National Science Foundation awards \#1925607, \#1629914.

\section{REFERENCES}

[1] Chao Huang, Chuxu Zhang, Jiashu Zhao, Xian Wu, Nitesh V Chawla, and Dawei Yin. 2019. MiST: A Multiview and Multimodal Spatial-Temporal Learning Framework for Citywide Abnormal Event Forecasting. In $W W W$.

[2] Huaxiu Yao, Chuxu Zhang, Ying Wei, Meng Jiang, Suhang Wang, Junzhou Huang, Nitesh V Chawla, and Zhenhui Li. 2020. Graph Few-Shot Learning via Knowledge Transfer. In $A A A I$.

[3] Lu Yu, Chuxu Zhang, Shangsong Liang, and Xiangliang Zhang. 2019. Multi-order Attentive Ranking Model for Sequential Recommendation. In AAAI.

[4] Lu Yu, Chuxu Zhang, Shichao Pei, Guolei Sun, and Xiangliang Zhang. 2018. Walkranker: A unified pairwise ranking model with multiple relations for item recommendation. In $A A A I$.

[5] Chuxu Zhang, Chao Huang, Lu Yu, Xiangliang Zhang, and Nitesh V Chawla. 2018. Camel: Content-aware and meta-path augmented metric learning for author identification. In $W W W$.

[6] Chuxu Zhang, Dongjin Song, Chao Huang, Ananthram Swami, and Nitesh V Chawla. 2019. Heterogeneous Graph Neural Network. In $K D D$.

[7] Chuxu Zhang, Dongjin Song, Chen Yuncong, Xinyang Feng, Cristian Lumezanu, Wei Cheng, Jingchao Ni, Bo Zong, Haifeng Chen, and Nitesh V Chawla. 2019. A deep neural network for unsupervised anomaly detection and diagnosis in multivariate time series data. In $A A A I$.

[8] Chuxu Zhang, Ananthram Swami, and Nitesh V Chawla. 2019. SHNE: Representation Learning for Semantic-Associated Heterogeneous Networks. In WSDM.

[9] Chuxu Zhang, Huaxiu Yao, Chao Huang, Meng Jiang, Zhenhui Li, and Nitesh V Chawla. 2020. Few-Shot Knowledge Graph Completion. In AAAI.

[10] Chuxu Zhang, Lu Yu, Yan Wang, Yan Wang, and Xiangliang Zhang. 2017. Collaborative User Network Embedding for Social Recommender Systems. In SDM.

[11] Chuxu Zhang, Lu Yu, Xiangliang Zhang, and Nitesh V Chawla. 2017. ImWalkMF: Joint matrix factorization and implicit walk integrative learning for recommendation. In IEEE Big Data.

[12] Chuxu Zhang, Lu Yu, Xiangliang Zhang, and Nitesh V Chawla. 2018. Task-guided and semantic-aware ranking for academic author-paper correlation inference. In IFCAI. 